\title{
Sub-ppb ammonia detection based on photoacoustic spectroscopy
}

\author{
Jean-Philippe Besson*, Stéphane Schilt, Luc Thévenaz, \\ Ecole Polytechnique Fédérale de Lausanne (EPFL), \\ Nanophotonics and Metrology Laboratory \\ CH-1015 Lausanne, Switzerland
}

\begin{abstract}
A photoacoustic sensor for the detection of ammonia using an EDFA-amplified semiconductor laser diode at $1532 \mathrm{~nm}$ is reported and for the first time a sub-ppb sensitivity of $0.6 \mathrm{ppb}$ is demonstrated in the near infrared.
\end{abstract}

Keywords: photoacoustic spectroscopy, trace gas sensing, diode laser, ammonia

\section{INTRODUCTION}

Sensitive and continuous ammonia $\left(\mathrm{NH}_{3}\right)$ monitoring is relevant in various applications, such as deNO $\mathrm{N}_{\mathrm{x}}$ processes widely used in power plants and incinerators to reduce $\mathrm{NO}_{\mathrm{x}}$ emissions [1], process control in the semiconductor industry, where extremely low levels of $\mathrm{NH}_{3}$ in clean rooms may drastically deteriorate the performances of lithography process [2], environment monitoring to check $\mathrm{NH}_{3}$ emissions from animal production facilities and automobile traffic [3] or in medicine to analyse breath $\mathrm{NH}_{3}$ levels as a diagnostic tool [4]. Whereas detection limits in the parts-per-million (ppm) or sub-ppm range are sufficient for some of these applications (i.e. deNO $\mathrm{N}_{\mathrm{x}}$ process), most of them require much better performances at parts-per-billions (ppb) level.

Photoacoustic spectroscopy (PAS) is a widely recognised method for its high performances in trace gas measurements from ppm down to ppb levels. This technique provides many advantages such as high selectivity and sensitivity, on-line, real time and contactless measurements in a compact experimental arrangement. It is based on the periodic absorption of a modulated laser beam tuned on an absorption line of the gas to be detected, so that the absorbed power induces a periodic heating and an acoustic wave is generated through normal thermal expansion. This acoustic wave is detected by a microphone and the signal amplitude is basically proportional to the gas concentration, to the laser power and to the strength of the absorption line. In addition, a configuration using semiconductor distributed feedback (DFB) lasers packaged for optical telecommunications makes the system compatible with fibre optics. This greatly facilitates the alignment of the setup and opens new perspectives toward ppb-level detection using fibre amplifiers.

We report a DFB laser-based photoacoustic sensor for ammonia traces detection at sub-ppm level and the first experimental demonstration that a sub-ppb level is actually reached using an erbium-doped fibre amplifier (EDFA) to improve the sensitivity.

\section{DESCRIPTION OF THE SETUP FOR PHOTOACOUSTIC SENSING}

Our photoacoustic (PA) sensor is used in a resonant configuration in which the first longitudinal acoustic mode is excited [5]. It is built out of stainless steel and consists in two buffer volumes and a central cylinder tube of radius $r=3 \mathrm{~mm}$ acting as an acoustic resonator (Figure 1a). This resonator has been carefully designed to optimize the photoacoustic response resulting from the combined interaction of light and sound. The length of the buffer volumes can be finely tuned in order to minimize the coupling of the ambient noise into the resonator. In addition, the entire PA cell is placed into an acoustic insulating housing that rejects the ambient noise. An optical telecommunications DFB diode laser emitting at $1532 \mathrm{~nm}$ has been selected for $\mathrm{NH}_{3}$ detection. The laser is fibre-pigtailed which facilitates the alignment of the system and makes possible an easy insertion of an EDFA to boost the optical power launched into the PA cell. This considerably improves the sensitivity, since the PA signal is directly proportional to the laser power. The output fibre is terminated by a built-in collimator directly mounted on the outer flange of the buffer volume.

*jean-philippe.besson@epfl.ch; phone +41216934772 ; fax +41216932614 
The first acoustic longitudinal mode is efficiently excited as the beam propagates along the axis of the resonator. The sound wave generated by the interaction of the light from the current-modulated laser and the gas molecules is detected by a sensitive electret microphone located at the centre of the resonator, i.e. at the maximum of the acoustic standing wave. A typical acoustic resonance curve obtained using this setup is shown in Figure 1b; an acoustic quality factor of $Q=28$ is reached with this resonator. The signal amplitude is measured using a lock-in amplifier with a time constant set to 10 s. Finally, an electronic module controls the laser modulation and processes the data.
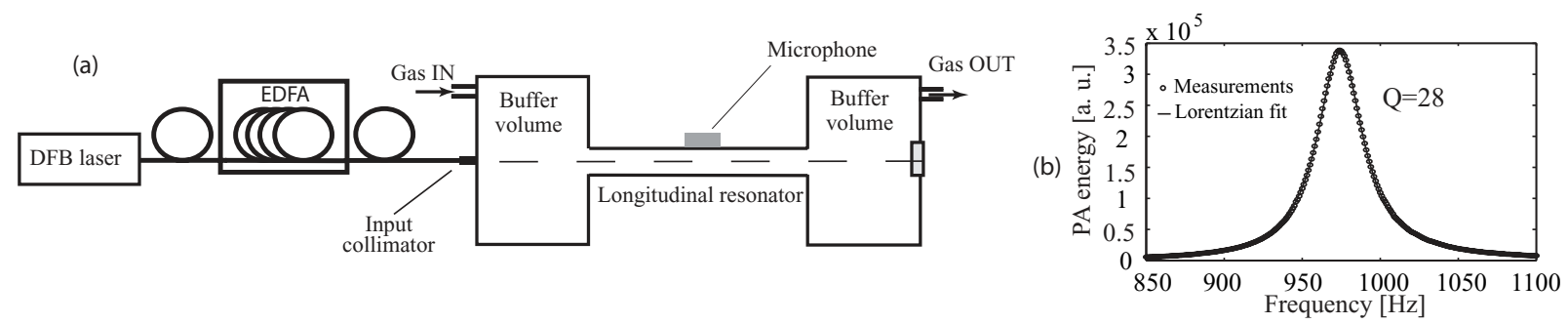

Fig. 1. (a) Schematic representation of the PA sensor based on a DFB laser and a PA cell operated in its first longitudinal mode. An EDFA can be inserted in order to increase the detection sensitivity (b) Acoustic resonance of the PA cell. Circles are experimental points and the curve is the result of a Lorentzian fit.

\section{EXPERIMENTAL RESULTS}

PA spectrum of ammonia has been measured over a few nanometres by tuning the laser temperature and by recording the generated PA signal (Figure 2). The strongest absorption observed at $1531.7 \mathrm{~nm}$ has been selected for $\mathrm{NH}_{3}$ traces detection. This absorption pattern is actually made of several overlapping lines [6] that cannot be resolved at atmospheric pressure. The response of the system to various ammonia concentrations was first measured without EDFA. Results are shown in Figure 3. The temperature of the laser was tuned to reach the proper absorption line and the current modulation amplitude was optimised to achieve the strongest PA signal. Different gas mixtures are obtained from certified

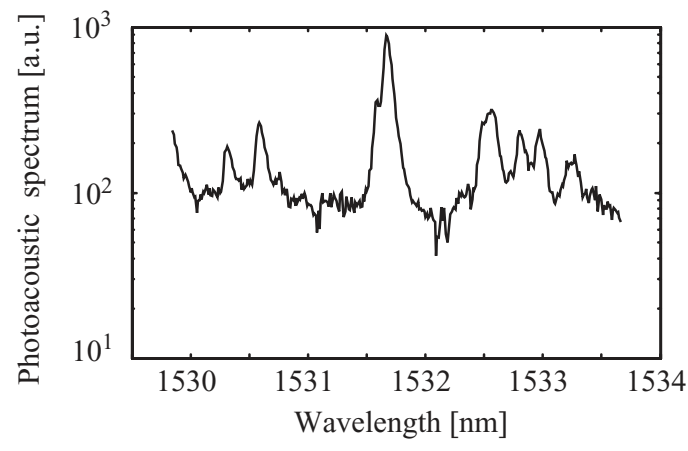

Fig. 2: Photoacoustic spectrum of $\mathrm{NH}_{3}$ measured using our experimental set-up. concentrations diluted with mass flow controllers. An excellent linearity was obtained and the ammonia concentration resulting in a noise-equivalent signal was found to be $44 \mathrm{ppb}$. The flow rate used in each measurement was 1 liter/min, which results in a fast response time without inducing additional acoustic noise. A higher rate would result in a turbulent flow and thus in a much increased noise level. The typical integration time of the lock-in amplifier was set to $10 \mathrm{~s}$.

(a)

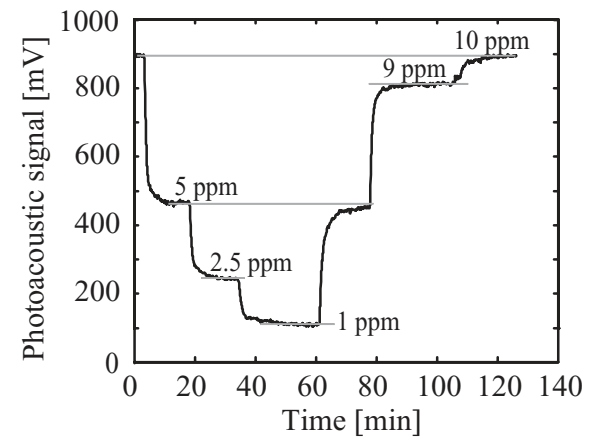

(b)

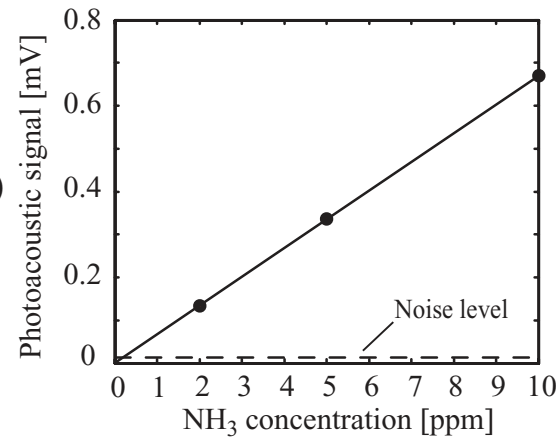

Fig. 3: (a) Response of the system to different $\mathrm{NH}_{3}$ concentrations; (b) Calibration curve. 
Light modulation required to generate the PA signal may be performed in two different ways using a DFB laser. Either an intensity modulation (IM) or a wavelength modulation (WM) may be predominant by modulating the laser injection current. In general, current modulation produces combined IM-WM, but two extreme situations may be encountered in which one type of modulation is strongly dominant. When the laser is square modulated from threshold to a large current, an on-off modulation of the optical power is achieved and IM is dominant. In a WM dominated regime obtained using a small current modulation (for both square and sinus modulation), the PA signal directly depends on the modulation depth and an optimum value may be determined (Fig. 4). In this latter case a residual IM is always nevertheless observed.

In the present situation, the optimum value is $I_{m o d}=18 \mathrm{~mA}$ for which the wavelength modulation is still dominant. The PA signal obtained when the laser is scanned through the ammonia absorption line shows like a derivative of the absorption line for a dominant wavelength modulation. This is no longer the case when intensity modulation is dominant [7] and the PA signal is directly proportional to the absorption line. This is clearly shown in the insets of Figure 4. The dip in the PA amplitude occurring for $I_{\text {mod }}=10 \mathrm{~mA}$ results from the shape of the $\mathrm{NH}_{3}$ absorption pattern which is not a single line, but is made of several (mainly two) overlapping lines. At low modulation depth the weak line on the left is clearly separated from the main absorption line and do not influence the main PA signal peak. As the modulation depth increases, the lines broaden and tend to overlap. Particularly at $I_{\text {mod }}=10 \mathrm{~mA}$, the out-of-phase response of the weaker line strongly contributes to the PA signal and cancels part of the in-phase signal from the stronger line. At higher modulation intensities, the broadening becomes dominant and the PA signal is mainly given by the strongest absorption line.

Then, the implementation of an EDFA was investigated as a tool to improve the detection sensitivity toward the ppb level. The EDFA was operated in the saturation regime and delivered around $750 \mathrm{~mW}$ of $1531.7 \mathrm{~nm}$ amplified power from the $18.5 \mathrm{~mW}$ laser, thus providing a $16 \mathrm{~dB}$ gain to the laser optical power. The total power at the output of the EDFA including amplified spontaneous emission was about $1 \mathrm{~W}$. In order to maximize the PA signal, a sinusoidal modulation was applied to the laser with amplitude similar to the optimum value defined in Figure 4 for a square modulation. The residual intensity modulation was measured and compared to the case of the non-amplified laser (Figure 5). The residual intensity modulation is an important parameter since it contributes to the window and wall noise. Using an optical amplifier in the saturation regime enables to reduce the IM index by a factor 10 . The use of the EDFA has the further advantage of maintaining a constant output power and thereof reducing the intensity modulation. This configuration therefore strongly increases the PA signal, but does not produce any additional window or wall noise.

a)

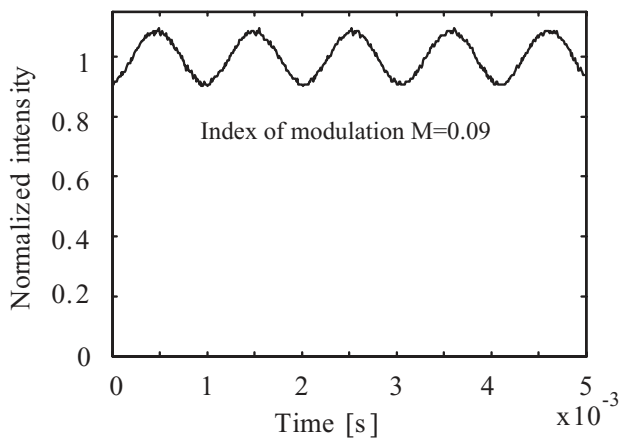

b)

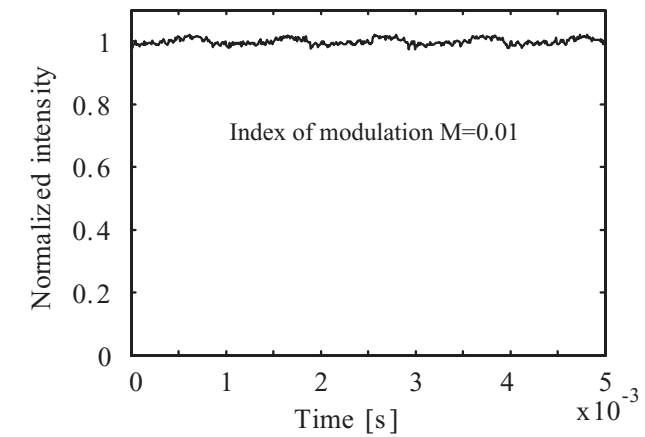

Fig 5: Residual intensity modulation a) with no EDFA b) with EDFA. The signals are normalized to the DC value 
The detection limit is evaluated by interpolating the concentration for a unity signal-to-noise ratio. Four different configurations were tested to determine the sensitivity of the system. A first set-up was configured with no optical amplifier and using a single pass of the laser beam through the cell. The second configuration was set up using a mirror at the end of the cell resulting in a double pass of the laser beam. Then these same two arrangements were tested after inserting the EDFA. In all cases the noise was recorded with a 10s integration time on the lock-in amplifier by slightly tuning the laser temperature, so that its emission lies off the absorption line. No special coating was used in the cell, which resulted in very long purging time due to adsorption and desorption processes [8] that did not allow to reach the noise level in the absorption line in a reasonable timescale. The results are summarized in Table 1.

\begin{tabular}{|l|c|c|c|c|}
\hline & Single pass & Double pass & Single pass EDFA & Double pass EDFA \\
\hline Output power $(\mathrm{mW})$ & 18.5 & 18.5 & 750 & 750 \\
\hline PA signal for $1 \mathrm{ppm} \mathrm{NH} \mathrm{NH}_{3}(\mathrm{mV})$ & 0.056 & 0.106 & 2.442 & 4.64 \\
\hline Noise level $(\mu \mathrm{V})$ & 2.49 & 2.52 & 2.73 & 2.63 \\
\hline Detection limit $(\mathrm{SNR}=1)(\mathrm{ppb})$ & $\mathbf{4 4}$ & $\mathbf{2 4}$ & $\mathbf{1}$ & $\mathbf{0 . 6}$ \\
\hline
\end{tabular}

Table 1: Performance of the sensor in different configurations.

A side positive benefit of the optical amplifier is that the noise level is kept constant at increased power levels, principally due to the fact that the frequency modulation is strongly dominant. As a consequence, the detection limit could be improved by a factor 40 enabling to reach the ppb level. A noise-equivalent detection limit of $0.6 \mathrm{ppb}$ was achieved which is to our knowledge the best sensitivity reached with near-infrared laser photoacoustic sensors.

\section{CONCLUSIONS}

The development of a high performance PA sensor has been demonstrated with a sub-ppm detection limit with nonamplified light, resulting from a thorough design oriented to optimize the photoacoustic response. The use of a tuneable DFB laser diode in combination with an optical amplifier improves considerably the sensitivity of the system and a detection limit of $0.6 \mathrm{ppb}$ of ammonia is demonstrated. These results are obtained with a $750 \mathrm{~mW}$ output power from an EDFA and a 10 s integration time. This clearly shows that compact and reliable ppb trace gas sensors operating in the near infrared using off-the-shelf fibre optics elements can be realized and that these sensors will play an important role in the future for the new challenges facing the developed societies.

\section{REFERENCES}

[1] G. Ramis, L. Yi and G. Busca, "Ammonia activation over catalysts for the selective catalytic reduction of $\mathrm{NO}_{x}$ and the selective catalytic oxidation of $\mathrm{NH}_{3}$. An FT-IR study", Catal. Today 28, 373-380 (1996).

[2] S. MacDonald, N. Clecak, R. Wendt, C. G. Willson, C. Snyder, C. Knors, N. Deyoe, J. Maltabes, J. Morrow, A. McGuire, S. Holmes, “ Airborne chemical contamination of a chemically amplified resist”, Proc SPIE 1466,2 (1991)

[3] S. Schilt, L. Thévenaz, M. Niklès, L. Emmenegger and C. Hüglin, "Ammonia monitoring at trace level using photoacoustic spectroscopy in industrial and environmental applications", Spectrochim. Acta A 60, pp. 3259-3268 (2004).

[4] L.R. Narasimhan, W. Gordon and C.K.M. Patel, "Correlation of breath ammonia with blood ures nitrogen and creatinine during hemodyalysis", PNAS 98, 4617-4621 (2001)

[5] J.-Ph. Besson, S. Schilt and L. Thévenaz, "Multi-gas sensing based on photoacoustic spectroscopy using tunable laser diodes", Spectrochim. Acta A 60, 3449-3456 (2004).

[6] M. E. Webber, D. S. Baer, and R. K. Hanson, "Ammonia monitoring near $1.5 \mu \mathrm{m}$ with diode-laser absorption sensors", Appl. Opt. 40 (12), 2031-2037, (2001).

[7] S. Schilt, Mesures de traces de gaz à l'aide de laser à semi-conducteur, $\mathrm{PhD}$ dissertation, Swiss Federal Institute of Technology, Lausanne, 2002

[8] J. Henningsen and N. Melander, "Sensitive measurement of adsorption dynamics with nonresonant phase photoacacoustics", Appl. Opt. 36 (27), 7037-7045, (1997). 